\title{
A DECOMPOSITION OF GALLAI MULTIGRAPHS
}

\author{
Alexander Halperin \\ Department of Mathematics \\ Lehigh University \\ Bethlehem, PA 18015, U.S.A. \\ e-mail: adh208@lehigh.edu \\ Colton Magnant \\ Department of Mathematical Sciences \\ Georgia Southern University \\ Statesboro, GA 30460, U.S.A.
}

e-mail: dr.colton.magnant@gmail.com

\author{
AND \\ KYLE PULA \\ Department of Mathematics \\ University of Denver \\ Denver, CO 80208, U.S.A. \\ e-mail: jpula@math.du.edu
}

\begin{abstract}
An edge-colored cycle is rainbow if its edges are colored with distinct colors. A Gallai (multi)graph is a simple, complete, edge-colored (multi)graph lacking rainbow triangles. As has been previously shown for Gallai graphs, we show that Gallai multigraphs admit a simple iterative construction. We then use this structure to prove Ramsey-type results within Gallai colorings. Moreover, we show that Gallai multigraphs give rise to a surprising and highly structured decomposition into directed trees.
\end{abstract}

Keywords: edge coloring, Gallai multigraph.

2010 Mathematics Subject Classification: 05C15. 
[1] B. Alexeev, On lengths of rainbow cycles, Electron. J. Combin. 13(1) (2006) \#105

[2] R.N. Ball, A. Pultr and P. Vojtěchovský, Colored graphs without colorful cycles, Combinatorica 27 (2007) 407-427. doi:10.1007/s00493-007-2224-6

[3] A. Diwan and D. Mubayi, Turán's theorem with colors, preprint, 2007.

[4] R.J. Faudree, R. Gould, M. Jacobson and C. Magnant, Ramsey numbers in rainbow triangle free colorings, Australas. J. Combin. 46 (2010) 269-284.

[5] A. Frieze and M. Krivelevich, On rainbow trees and cycles, Electron. J. Combin. 15 (2008) \#59.

[6] S. Fujita and C. Magnant, Extensions of Gallai-Ramsey results, J. Graph Theory 70 (2012) 404-426. doi:10.1002/jgt.20622

[7] S. Fujita and C. Magnant, Gallai-Ramsey numbers for cycles, Discrete Math. 311 (2011) $1247-1254$. doi:10.1016/j.disc.2009.11.004

[8] S. Fujita, C. Magnant and K. Ozeki, Rainbow generalizations of Ramsey theory: a survey, Graphs Combin. 26 (2010) 1-30. doi:10.1007/s00373-010-0891-3

[9] S. Fujita, C. Magnant and K. Ozeki. Rainbow generalizations of Ramsey theory: a survey, (2011) updated. http://math.georgiasouthern.edu/ cmagnant

[10] T. Gallai, Transitiv orientierbare Graphen, Acta Math. Acad. Sci. Hungar. 18 (1967) 25-66. doi:10.1007/BF02020961

[11] A. Gyárfás, G. Sárközy, A. Sebő and S. Selkow, Ramsey-type results for Gallai colorings, J. Graph Theory 64 (2010) 233-243.

[12] A. Gyárfás and G. Simonyi, Edge colorings of complete graphs without tricolored triangles, J. Graph Theory 46 (2004) 211-216. doi:10.1002/jgt.20001

[13] P. Vojtěchovský, Periods in missing lengths of rainbow cycles, J. Graph Theory $6 \mathbf{6}$ (2009) 98-110. doi:10.1002/jgt.20371

Received 28 November 2012

Revised 9 April 2013

Accepted 9 April 2013 